\title{
Bei allergischer Rhinitis auch an Augensymptome denken
}

_ Für die Behandlung der allergischen nitis and its Impact on Asthma)-Leitlinien bei allen Schwergraden der Erkrankung den Einsatz intranasaler Steroide (INS). Fluticasonfuroat (Avamys ${ }^{\circledR}$ ) habe von allen verfügbaren INS die höchste Affinität zum Glukokortikoidrezeptor und eine besonders lange Verweildauer im Nasengewebe, so Dr. Bettina Hauswald, Dresden. Das gewährleiste eine 24-Stunden-Wirkung. Außerdem werde der Wirkstoff schon in der Nase vollständig abgebaut, sodass keine systemischen Nebenwirkungen zu befürchten seien. Fluticasonfuroat verbessert nicht nur die nasalen Symptome der allergischen Rhinitis, sondern auch die okulären Symptome, unter denen mindestens drei Viertel der Patienten leiden.

Eine aktuelle Metaanalyse von 35 placebokontrollierten Studien bei saisonaler allergischer Rhinitis (SAR) hat ergeben, dass nur Fluticasonfuroat eine konsistent positive Wirkung auf die Augensymptome hat. Bei anderen INS hingegen waren die Effekte tete Dr. Oliver Pfaar, Wiesbaden. Weitere Rhinitis empfehlen die ARIA (Allergic Rhiwidersprüchlich und inkonsistent, berich-

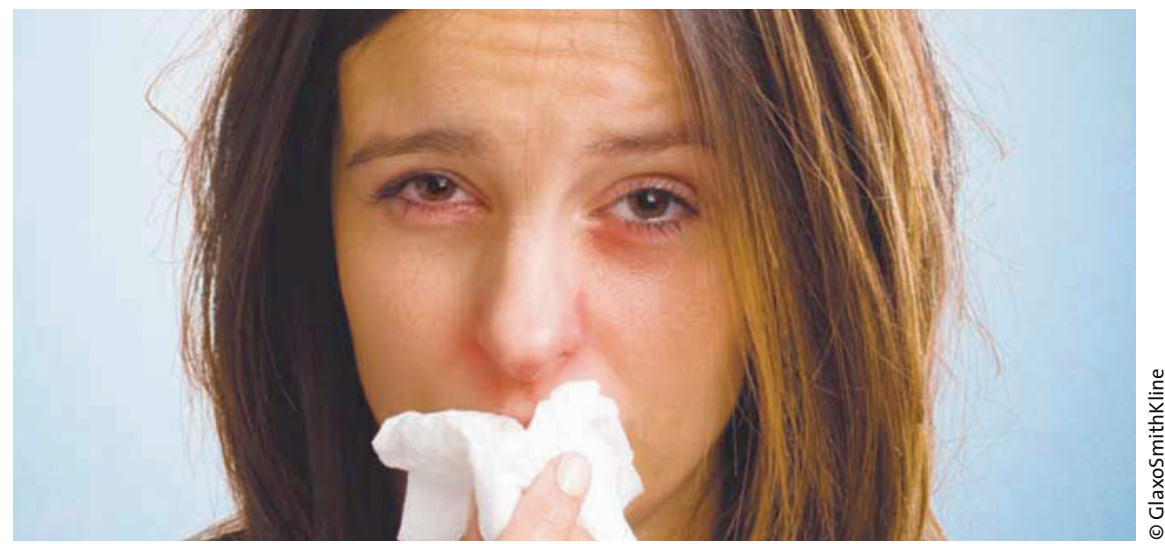

Mindestens drei Viertel der Patienten mit allergischer Rhinits leiden auch unter Augensymptomen.
SAR-Studien mit Fluticasonfuroat haben gezeigt, dass sich neben der Symptomatik auch alle untersuchten Domänen der Lebensqualität von Patienten mit allergischer Rhinitis signifikant und konsistent verbesserten.

Die Therapie mit Fluticasonfuroat ist auch aus Kostenaspekten effektiv. Anfang Februar 2010 wurde der Preis um 25\% reduziert, sodass nun ein größerer Verordnungsspielraum ermöglicht wurde.

aam

Pressegespräch „Start der Allergiesaison: Nasen- und Augensymptome jetzt schon wirksam und kostengünstig mit Avamys ${ }^{\circledR}$ behandeln". München, 23. Februar 2010 Veranstalter: GlaxoSmithKline, München

\section{Neue Hoffnung für Schnarcher und Schlafapnoe-Patienten}

— Während die Therapie des Scharchens aus einem breiten Spektrum an konservativen Maßnahmen bis hin zu verschiedenen operativen Möglichkeiten besteht, ist der Goldstandard für das schwere obstruktive Schlafapnoesyndrom die CPAP (Continuous Positive Airway Pressure)-Therapie. Daneben gibt es eine Reihe von „mechanischen Schnarchhilfen", die nun um die Applikation Velumount $^{\odot}$ erweitert wurde. Diese
„Schnarchspange“ wird durch geschultes Fachpersonal individuell angepasst, wobei die Velumount-Methode in Deutschland unter der Obhut von HNO-Ärzten steht. Zur Beurteilung der Wirksamkeit von Velumount $^{\odot}$ liegen derzeit zwei Studien aus der Schweiz vor. So tolerierten in einer Studie mit 39 Schlafapnoe-Patienten, die mit der Spange schliefen, 22 den Draht im Mund. Bei 21 nahmen das Schnarchen und die subjektive

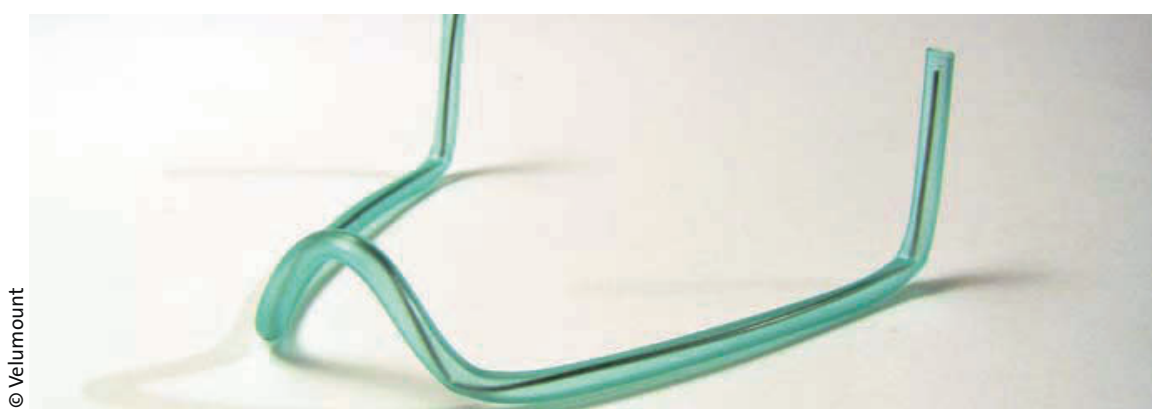

Schläfrigkeit während des Tages deutlich ab. In einer zweiten Studie wurden insgesamt 37 Patienten mit und ohne Velumount ${ }^{\odot}$ mittels eines Fragebogens (Epworth Sleepiness Scale), einer Polygrafie und nächtlicher Ösopahgus-Druckmessung (Manometrie) untersucht. Die Punktezahl im Fragebogen nahm dabei im Mittel von 8,2 auf 3,9 ab, der Ösophagusdruck ließ sich von $15 \mathrm{~cm}$ auf $11,4 \mathrm{~cm}$ $\mathrm{H}_{2} \mathrm{O}$ reduzieren. Der Apnoe-Hypopnoeindex der 22 Patienten mit Schlafapnoe reduzierte sich durch das Tragen der Schnarchspange von 23,6 auf 14 .

In der Zusammenschau bedeutet diese neuartige Methode bei richtiger Indikation für die Betroffenen ein wirksames Hilfsmittel zur Reduktion von Scharchen und Atempausen im Schlaf.

Nach Informationen von Velumount, Augsburg 